\title{
Korean Guideline for the diagnosis and treatment of onychomycosis: A Consensus Update by Korean Society for Medical Mycology
}

\author{
J. Park, J.H. Nam, J.H. Lee, J.S. Park, J.H. Mun, Y.W. Lee, J.S. Choi, M.K. Suh, K.H. Kim, W.J. Lee, J.B. Lee, H.C. Ko, H.J. Kim \\ Executive Committee of Onychomycosis Guideline of Korean Society for Medical Mycology
}

Background: Since the treatment guidelines for onychomycosis have been released by organization from different countries, there have been several advances in management of onychomycosis.

Objectives: The executive committee for treatment guideline of onychomycosis of in Korean society for medical mycology is aimed to provide an up-to-date, practical, algorithmic guideline for the management of onychomycosis in Koreans.

Methods: We reviewed thoroughly relevant literatures. Then, the structured algorithm was developed through a full discussion, robust agreement between members, and, ultimately, voting on the conflicting subjects.

Results: The presence of clinical signs such as yellow discoloration or thickening of the nail, onycholysis, is highly predictive; however, mycological examination should be done to confirm clinical suspicion of onychomycosis and rule out other nail disorders before starting the treatment. Various therapeutic options are available for onychomycosis and the mainstays of the therapy are topical and systemic antifungal agents. Currently, there are available antifungal agents in Korea that include oral terbinafine, itraconazole, fluconazole and topical efinaconzole, ciclopirox, amorolfine. Factors that may influence the selection of topical versus systemic therapy are the nail variables (clinical subtype, disease severity), patients' variables (underlying medical illness, drug-drug interactions).
For mild cases, both systemic and topical therapy can be an option, whereas systemic therapy is a treatment choice for moderate-to-severe cases if there are no contraindications to systemic antifungal agents. Oral terbinafine is the first-line medication for dermatophyte infection; however, it may be changed depending on the causative fungi. Combination treatment of topical and systemic antifungal agents is recommended to increased efficacy in severe cases. Adjuvant treatments, physical or chemical removal of the involved nail, are typically reserved for patients who cannot be successfully treated with medical treatment alone. Alternative treatments includes laser therapy ( $\mathrm{Nd}$ :Yag or diode laser). They can be performed when standard medical treatments cannot be applicable regardless of the severity of onychomycosis. Currently, lasers are only approved for temporary cosmetic improvement of the nail on onychomycosis. During the treatment period, proper evaluation of treatment response at 3, 6, 12, 18 months after the completion of the treatment should be rendered. After a patient has achieved effective cure, patient's education for prevention is recommended to minimize reinfection or recurrence.

Conclusion: The Korean consensus guideline for onychomycosis is designed to promote the assessment and treatment of onychomycosis.

Figure 1. Proposed algorithm for the diagnosis and treatment of onychomycosis

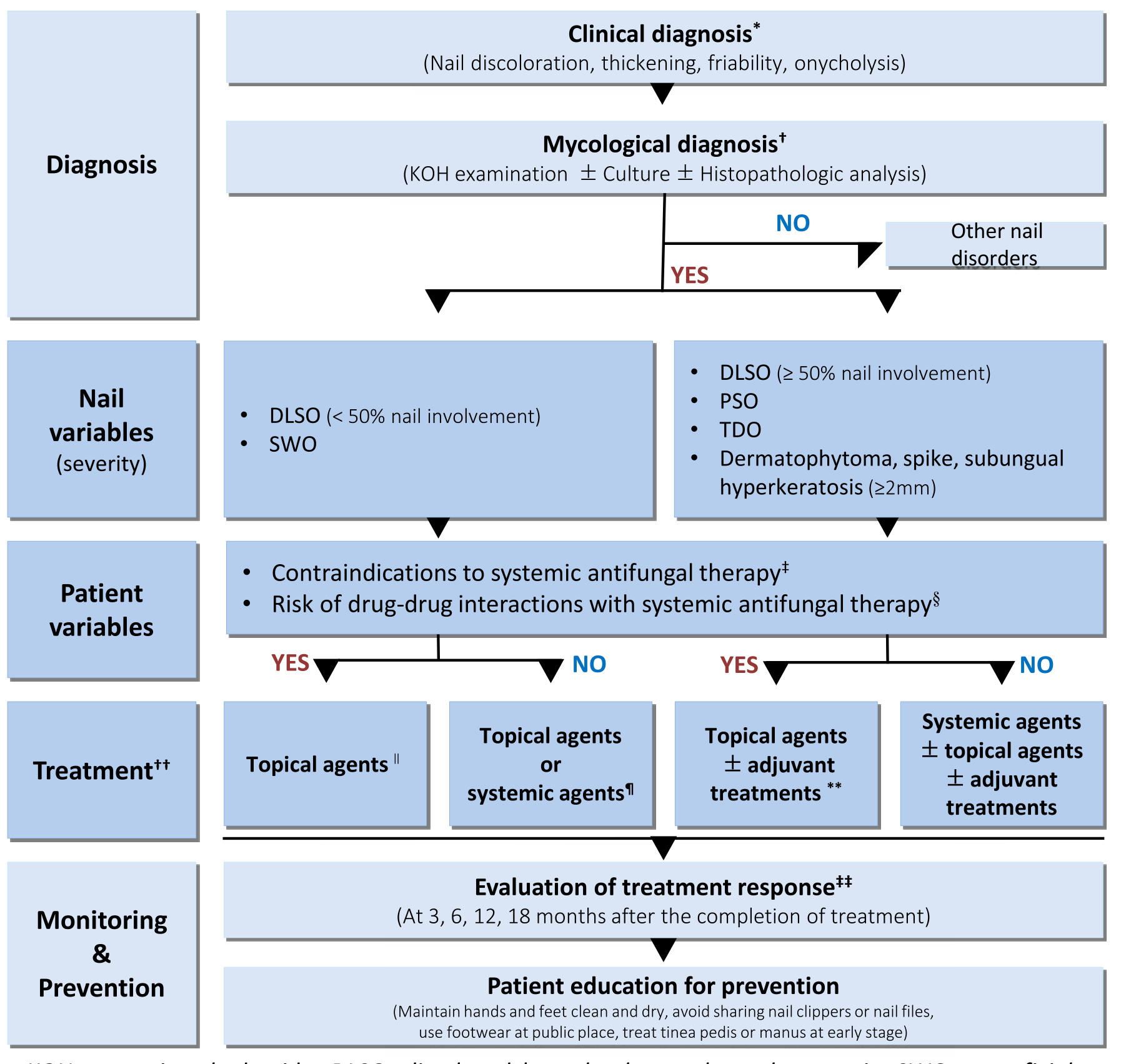

$\mathrm{KOH}$, potassium hydroxide; DLSO, distal and lateral subungual onychomycosis; SWO, superficial white onychomycosis; PSO, proximal subungual onychomycosis; TDO, total dystrophy onychomycosis

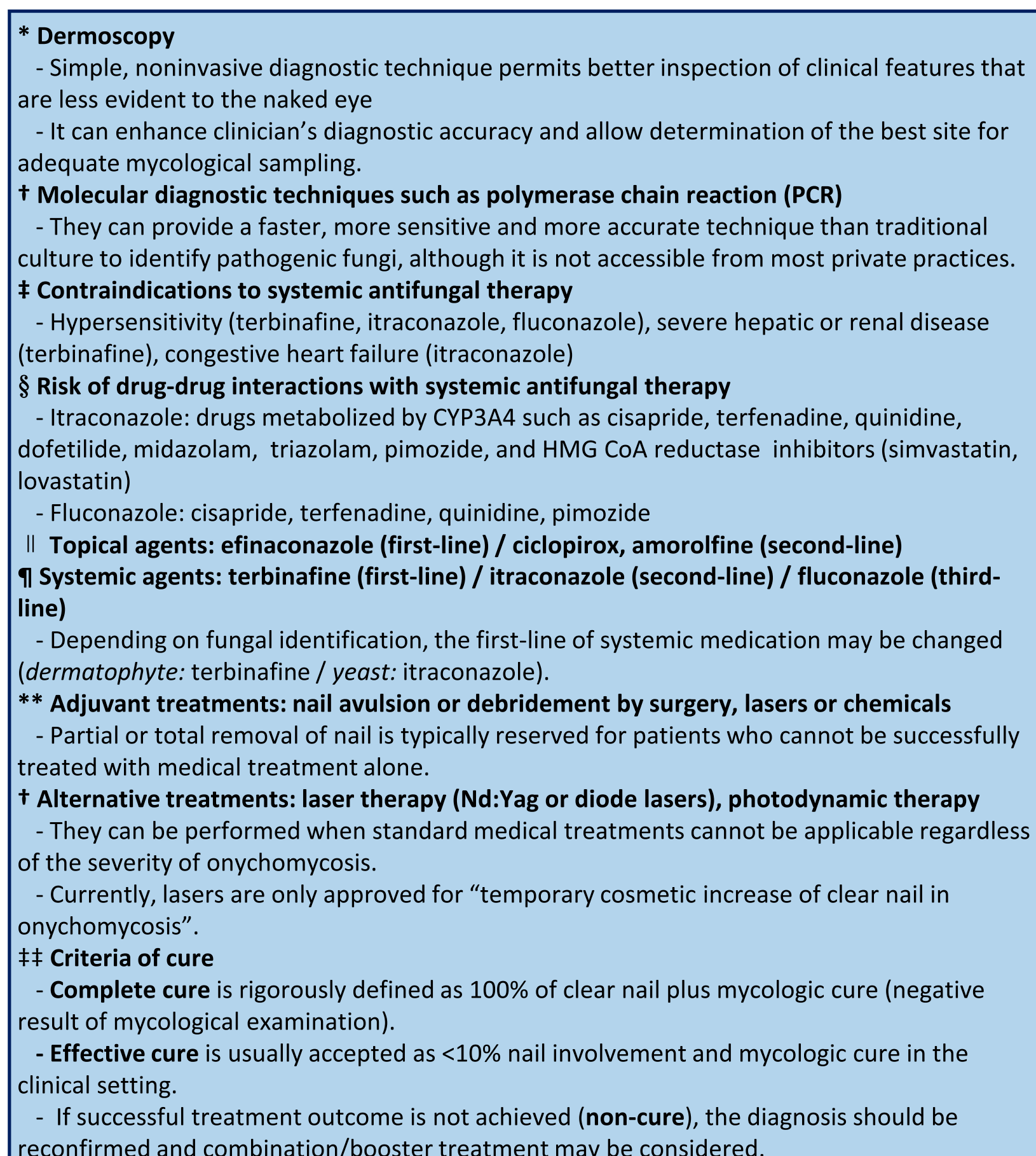

econfirmed and combination/booster treatment may be considered. 